\title{
Condiciones de seguridad alimentaria en una comunidad indígena de Colombia
}

\author{
I ${ }^{1}$ Juan Camilo Calderón Farfán, ${ }^{2}$ Dolly Arias Torres, ${ }^{3}$ Martha Nidia Papamija Gómez, \\ ${ }^{4}$ Maria Paula Quintero Tamayo I
}

Resumen: La seguridad alimentaria se consigue cuando todas las personas, en todo momento, tienen acceso físico y económico a suficiente alimento, seguro y nutritivo, para satisfacer sus necesidades alimenticias y sus preferencias, con el objeto de llevar una vida activa y sana. Objetivo: Explorar condiciones de seguridad alimentaria en los comuneros del Resguardo Indígena La Gabriela.

Método: Estudio cualitativo, de alcance exploratorio, que aplicó las técnicas de foto-voz y grupos de discusión. Se efectuó análisis de contenido. Resultados: Las condiciones de seguridad alimentaria de la comunidad indígena La Gabriela están asociadas al territorio y a prácticas alimentarias modificadas por influencia externa, lo cual afecta su autonomía generando dependencia a cadenas comerciales estimuladas por el libre mercado. Es preciso formular políticas que fortalezcan las creencias, costumbres y tradiciones ancestrales, lo cual requiere mayor atención por parte de las entidades estatales y los gobiernos.

> Palabras clave: Seguridad alimentaria; condiciones; alimentación; comunidad indígena; producción de alimentos.

\author{
1 Universidad Surcolombiana, \\ Facultad de Salud, Colombia \\ (juan.calderon@usco.edu.co). \\ ORCID: 0000-0001-5965-5771 \\ ${ }^{2}$ Universidad Surcolombiana, \\ Facultad de Salud, Colombia \\ (dolaria@usco.edu.co). \\ ORCID: 0000-0002-4409-2424 \\ ${ }^{3}$ Universidad Surcolombiana, \\ Facultad de Salud, Colombia \\ (marthagoetz@hotmail.com). \\ ORCID: 0000-0003-0866-6408 \\ ${ }^{4}$ Universidad Surcolombiana, \\ Facultad de Salud, Colombia \\ (mariapaulaqt@live.com) \\ ORCID: 0000-0001-5856-0885
}

Recebido em: 08/03/2018 Aprovado em: 09/08/2018 Revisado em: 14/09/2018 


\section{Introducción}

El primer concepto de seguridad alimentaria se desarrolla en 1966, en el marco de la Cumbre Mundial de la Alimentación (CMA), dirigida por la Organización de las Naciones Unidas para la Agricultura y la Alimentación (FAO). Allí se definió que:

[...] la seguridad alimentaria a nivel de individuo, hogar, nación y global, se consigue cuando todas las personas, en todo momento, tienen acceso físico y económico a suficiente alimento, seguro y nutritivo, para satisfacer sus necesidades alimenticias y sus preferencias, con el objeto de llevar una vida activa y sana (FAO, 2014).

En 1974, la Seguridad Alimentaria Nacional (SAN), se centra en la producción y disponibilidad de suministros alimentarios suficientes a escala de un país. En la década de los 80 , se adiciona el acceso económico físico a los alimentos por parte de las familias pobres, idea que contribuyó decisivamente en la teoría de las titularidades de Amartya Sen. Después se integran estos dos conceptos, mismos que se complementan con la aparición de nuevos enfoques explicativos que permiten un abordaje integral y complejo del fenómeno.

El abordaje integral de la SAN incluye el análisis de los determinantes económicos de ingresos, conocimientos e información sobre el valor cultural de los alimentos, la salud y su relación con la nutrición, la seguridad alimentaria y los derechos legales. También se reconoce lo nutricional con sus factores condicionantes y la interacción entre estas dos categorías. Finalmente se incorpora la inocuidad de los alimentos (y las preferencias culturales) y se reafirma la Seguridad Alimentaria como un derecho humano, definiéndose como Seguridad Alimentaria y Nutricional (FAO; DEPARTAMENTO, 2015).

En el Plan Nacional de Seguridad Alimentaria y Nutricional (PNSAN) 20122019, se establece que:

La Seguridad alimentaria y nutricional es la disponibilidad suficiente y estable de alimentos, el acceso y el consumo oportuno y permanente de los mismos en cantidad, calidad e inocuidad por parte de todas las personas, bajo condiciones que permitan su adecuada utilización biológica, para llevar una vida saludable y activa. (REPÚBLICA DE COLOMBIA; CONPES, 2008).

El objetivo es ofertar los alimentos necesarios para el mejoramiento de la situación alimentaria y nutricional de toda la población colombiana. La seguridad alimentaria como derecho exige a los Estados y organizaciones internacionales, el desarrollo de programas y acciones para erradicar el hambre. Ejemplo de estas iniciativas son 
los Objetivos de Desarrollo del Milenio (NACIONES UNIDAS, 2015) y actuales Objetivos de Desarrollo Sostenible (ODS), que establecen como meta para el 2030, poner fin a todas las formas de malnutrición, incluso logrando, a más tardar en 2025, las metas convenidas internacionalmente sobre el retraso del crecimiento y la emaciación de los niños menores de 5 años (MORAN, 2015).

Pese a los desarrollos teóricos sobre SAN, la inseguridad en este campo es un problema común y creciente en el mundo, que afecta a los grupos más vulnerables, de manera particular a los indígenas (PEÑA-VENEGAS et al., 2009). Estudios latinoamericanos presentan altas prevalencias de desnutrición y anemia frente a la población no indígena (BARRETO et al., 2014; COIMBRA JR; COIMBRA, 2014; TELLO; MIRANDA; ESPINOZA, 2006; ANTICONA; GAVIDIA; TELLO, 2008; RAMOS et al., 2002).

En Colombia, la Encuesta Nacional de la Situación nutricional (ENSIN) del 2010, visibiliza la inequidad en seguridad alimentaria de las comunidades indígenas, expresada en retraso en talla, deficiencia de hierro, vitamina A y Zinc en menores de cinco años, y anemia en mujeres gestantes, mayores a las presentadas en población no indígena (ARIAS et al., 2013; FAO; DEPARTAMENTO, 2015).

En el departamento del Huila, la desnutrición crónica en niños indígenas menores de cinco años fue de 44,7\% para el 2010(IPS-I CRIHU, 2013), información que evidencia una problemática en materia de seguridad alimentaria y nutricional, con limitaciones de información detallada acerca de la realidad de cada uno de los pueblos y las condiciones que la determinan, lo que amerita su investigación e intervención.

Con base en lo expuesto en párrafos precedentes, se formuló el siguiente interrogante: ¿Cuáles son las condiciones de seguridad alimentaria de la comunidad indígena Tama - Páez la Gabriela, Neiva, Huila, Colombia? El estudio tuvo como objetivo explorar las condiciones de Seguridad Alimentaria en el Resguardo indígena La Gabriela, ubicado en la ciudad de Neiva, para identificar realidades susceptibles de transformación a nivel comunitario.

\section{Materiales y métodos}

Estudio cualitativo de alcance exploratorio. Población conformada por 321 habitantes, organizados en 48 familias, pertenecientes al Resguardo indígena La Gabriela, ubicado a una distancia aproximada a $10 \mathrm{~km}$ de la ciudad de Neiva (Capital del Departamento del Huila, Colombia). 
La muestra la integraron 18 personas, escogidas por la misma comunidad en Asamblea, conforme a la dinámica organizativa del Resguardo, mediante muestreo por criterio (QUINN, 2002). Entre los participantes se encontraban: tres sabedores ancestrales, un gobernador, un docente, cuatro madres, cuatro jóvenes, dos adultos mayores, dos guardias indígenas y el coordinador del Programa de salud. Los seleccionados fueron mayores de 14 años de edad, con permanencia mayor a dos años en el Resguardo.

Para la recolección de información se utilizaron las técnicas de foto-voz (WANG; BURRIS, 1994) y grupos de discusión (HUERTAS; VIGIER, 2010; CALLEJO, 2009). Previo a la aplicación de las mismas, se dieron a conocer los objetivos del estudio, se brindó capacitación acerca del manejo de cámaras fotográficas y captura de imágenes representativas sobre el objeto de estudio. En los grupos de discusión se indagó acerca de las condiciones de seguridad alimentaria (Acceso, disponibilidad, consumo, aprovechamiento biológico y calidad e inocuidad). Posteriormente se realizaron foto-sesiones y socialización explicativa de las mismas a partir de narrativas (WANG; BURRIS, 1994); éstas fueron grabadas y transcritas para análisis de contenido (AMEZCUA; TORO, 2002), identificando frases significativas que se agruparon por temas y subtemas emergentes. Al finalizar, se socializaron los resultados con el grupo de estudio para la validación final.

\section{Consideraciones éticas del estudio}

El estudio fue clasificado sin riesgo según Resolución n. 8430/1993, del Ministerio de Salud de Colombia (MINISTERIO..., 1993). Se tuvieron en cuenta los principios éticos de investigación en seres humanos (CIOMS, 2002), de la Organización Mundial de la Salud, así como los postulados éticos de la investigación en comunidades indígenas (BRANT, 2004). El proyecto contó con el asentimiento del Resguardo indígena y el aval del Comité de Ética de la Facultad de Salud de la Universidad Surcolombiana mediante sesión del día 03 de marzo de 2017.

\section{Resultados}

Para el análisis de resultados se tuvieron en cuentan las características que denotan la SA: "disponibilidad y acceso", "consumo", "aprovechamiento", y "calidad e inocuidad de los alimentos". 
En relación con la disponibilidad y acceso a los alimentos, se encontró influencia de las condiciones asociadas a tenencia, extensión y uso de la tierra; resaltándose la aridez de los suelos del territorio, la insuficiencia de los recursos hídricos y las condiciones climáticas adversas. Todas ellas ocasionan pérdida de especies vegetales y animales usadas tradicionalmente como fuente de alimentación. Algunos fragmentos al respecto:

El área que nosotros tenemos es mínima en la extensión del resguardo; aunque en las escrituras coloniales era de doce mil hectáreas, ahora solo tenemos quinientas cincuenta y ocho. (Grupo de Discusión 1. Hombre Comunero).

En épocas de lluvia, cosechamos maíz, ahuyama y yuca, en verano nada se da. En verano, las personas que tienen aljibes les dura por más tiempo el agua. (Grupo de Discusión 1. Mujer Comunera).

El Rio Arenoso tenía pescado, había cucha, mojarra, zapateros, mochos y mojarra negra, ahora ya no se ve nada de eso, ya se perdió. (...) Antes por el Arenoso no había carretera; ahora hay vías hasta Cerro Neiva; cuando la construyeron tumbaron la arborización, ahora el arenoso es seco, se perdió todo, se rompió el equilibrio de la naturaleza. (Grupo de Discusión 1. Gobernador).

La disponibilidad y el acceso a los alimentos, también es afectada por factores externos a la comunidad; entre ellos, invasión territorial del resguardo por población diferente a la indígena, prácticas de explotación de recursos naturales, construcción de obras urbanísticas como carreteras y planes de vivienda, ingreso de prácticas foráneas de producción de alimentos con utilización de abonos tipo agroquímicos y semillas transgénicas. Enseguida se consignan expresiones alusivas:

Las construcciones se extienden desde Neiva hacia nuestro resguardo invadiendo terrenos coloniales. Y eso que existe una ley que prohíbe construir a cinco kilómetros de los asentamientos nuestros; no más miren a la salida de aquí, están Bosques de San Luis y otras construcciones (...) Otro asunto que limita nuestros espacios físicos son las exploraciones de petróleo, ubicadas muy cerca a nuestra comunidad. (Grupo de Discusión 1. Hombre Comunero).

El maíz de semilla propia ya se perdió, ahora ya ese maíz es con transgénicos, y es un maíz diferente; desde ahí comienza a afectarnos porque si usted no le echa abono de afuera ese maíz no va a producir. (Grupo de Discusión 1. Hombre Comunero).

Las prácticas de consumo de alimentos están determinadas por condiciones naturales y de comercialización. En el resguardo hay necesidad de abastecer a las familias con alimentos producidos fuera de la comunidad, que en su mayoría utilizan químicos en su producción, para lo cual siembran productos para la venta, como la caña de azúcar. El cultivo de la caña de azúcar se caracteriza por resistir largos periodos de sequía. 
Figura 1. Producción de panela

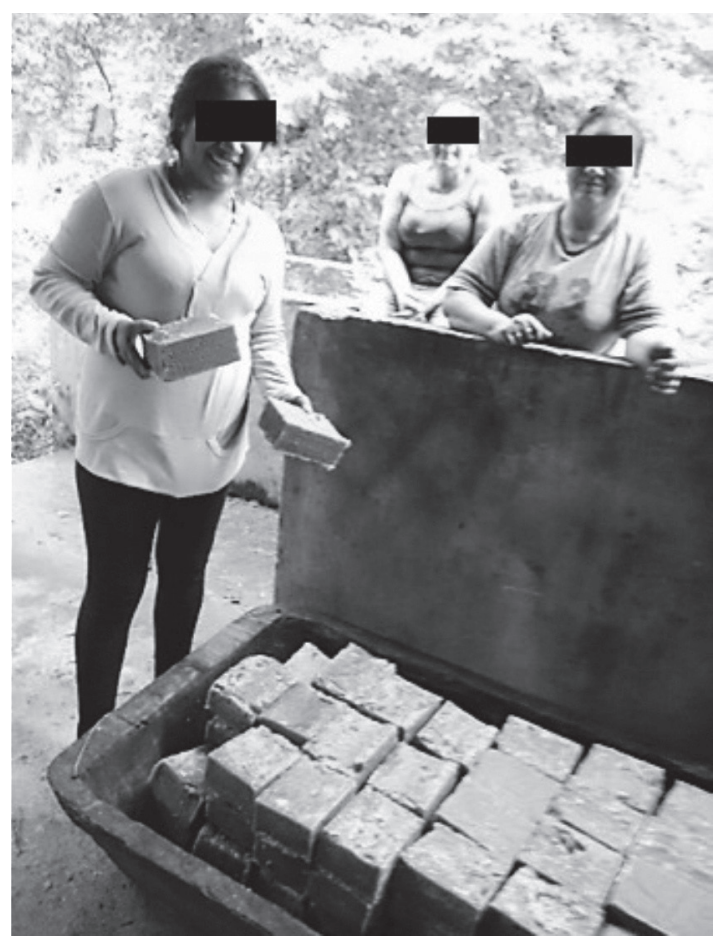

Nosotros sacamos panela, la vendemos; y con esa plata hacemos un mercado que repartimos a todas las familias. (Fotosesión 1, Mujer Comunera).

El plátano, el maíz y la yuca los tenemos acá. Parte de esos productos los vendemos para comprar alimentos que no producimos como el arroz, el azúcar, o la cebolla y la papa que no se dan aquí. (Fotosesion1, Mujer Comunera)

La comunidad identifica la influencia de los medios de comunicación en la población más joven del Resguardo, que incita al consumo de alimentos industrializados:

Hoy, en muchos programas se estimula la adopción de hábitos alimenticios diferentes a los acostumbrados; a través de la televisión se provoca el consumo de dulces, chitos, papas fritas, etc.; todo lo comercializan, es el desarrollo comercial de un mundo que va a toda velocidad, no para. Antes no se veía eso; están cambiando nuestra cultura a través de internet, televisión y otros medios; lo más triste es que es muy difícil controlar eso. (Fotosesión 2, adulto mayor).

Hoy los niños prefieren dulces a la comida casera; eligen comidas chatarra. Cambian un jugo natural o un caldo de alimento por una gaseosa (Fotosesión 3, madre). 
La utilización y aprovechamiento de los alimentos la relacionan con conceptos de buena alimentación asociada a buena salud y nutrición; en contraste, la mala alimentación, se presenta cuando se consumen alimentos producidos por procesos de industrialización, que pueden generar enfermedades y muerte. Emergen dos categorías, la alimentación propia y la foránea.

\section{Figura 2. Lactancia materna}

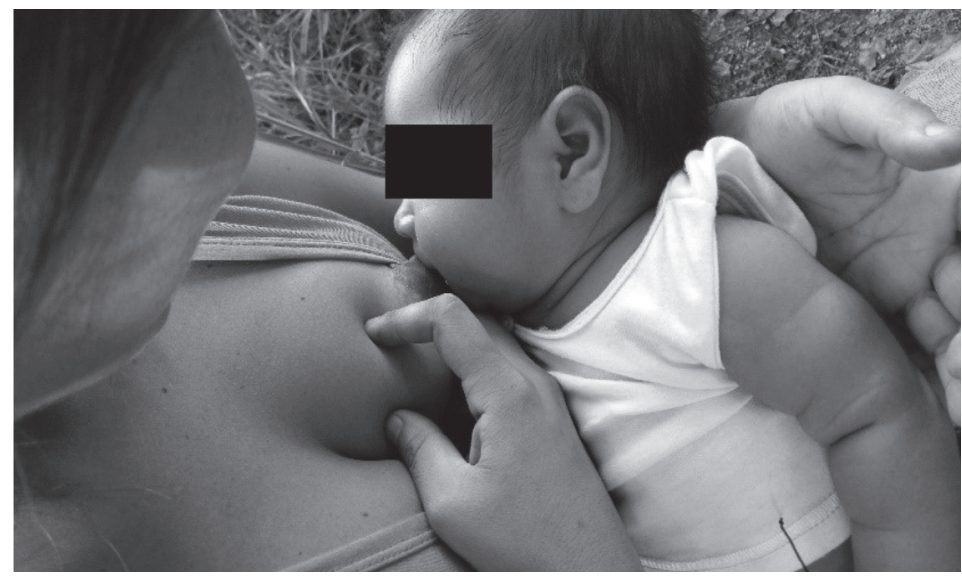

Aquí le tomamos la foto a un hijo de una compañera que está lactando; el niño está recibiendo buena alimentación porque la lactancia es lo mejor, lo primordial que hay para un bebé. (Fotosesión 2, madre).

Figura 3. Adulto mayor de la comunidad

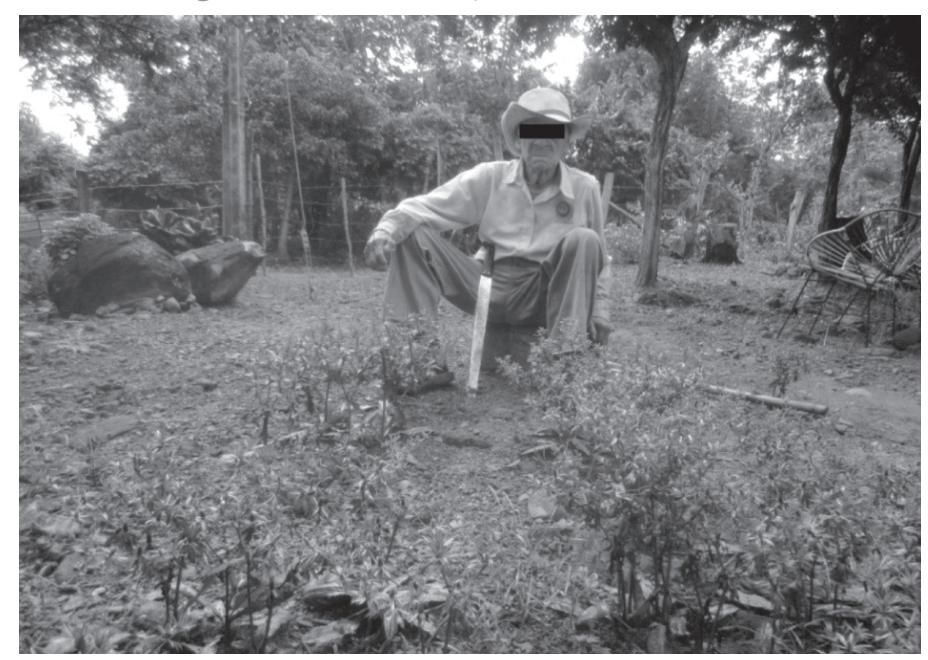


Este es un abuelo de 97 ańos, que goza de buena salud, según él porque se alimentaba muy bien, con carne de monte (Fotosesión 2, madre).

Por último, la categoría de calidad e inocuidad de los alimentos, analizada desde las condiciones del territorio, muestra la relevancia de la utilización de productos orgánicos y libres de agroquímicos, como factor determinante de la calidad del mismo. Por tanto, la conservación de prácticas tradicionales y recursos naturales de producción de alimentos, se posiciona como el principal factor que garantiza la calidad e inocuidad de los alimentos que se consumen. Este proceso se constituye en práctica de resistencia frente a las tendencias industriales de producción de alimentos de influencia externa, caracterizados por mayor producción en menor tiempo.

\section{Figura 4. Cultivo de plátano y yuca}

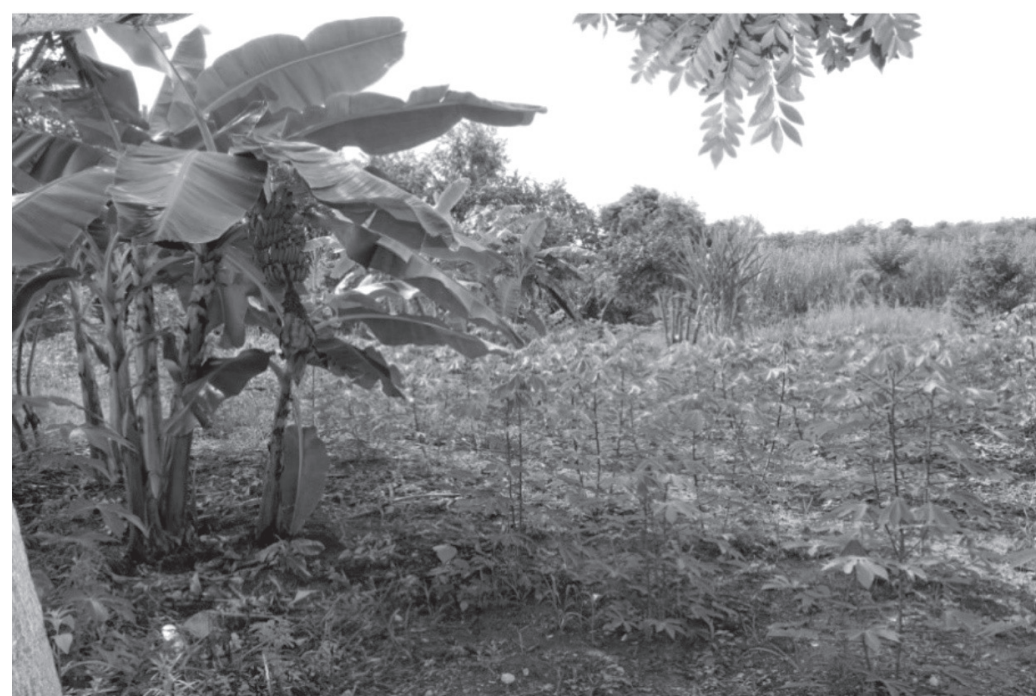

Todo lo que se ve en la foto, los bandos, la yuca y otros productos son naturales; aquí utilizamos abono orgánico, lo que da la tierrita. El abono orgánico que se prepara es el estiércol de hormiga, la tierra negra que bota el árbol y esa hoja que se pudre, se revuelve con otra tierra y con arena; ese es el abono propio; pero a veces tenemos que usar abonos comerciales. (Fotosesión 1. Mujer Comunera).

La comunidad utiliza modelos agropecuarios externos como la producción de huevos y la crianza de pollos de engorde, como fuente de ingresos para complementar la canasta familiar. Esta producción adquiere un carácter particular y diferenciado; por ejemplo, los pollos destinados para la venta son alimentados con concentrados, 
dado que su producción es más rápida; por el contrario, los pollos para el consumo interno se crían "sueltos en el patio" para que se alimenten de la tierra, de desperdicios de la cocina, etc.

\section{Figura 5. Pollos para la venta vs Pollos para el consumo}

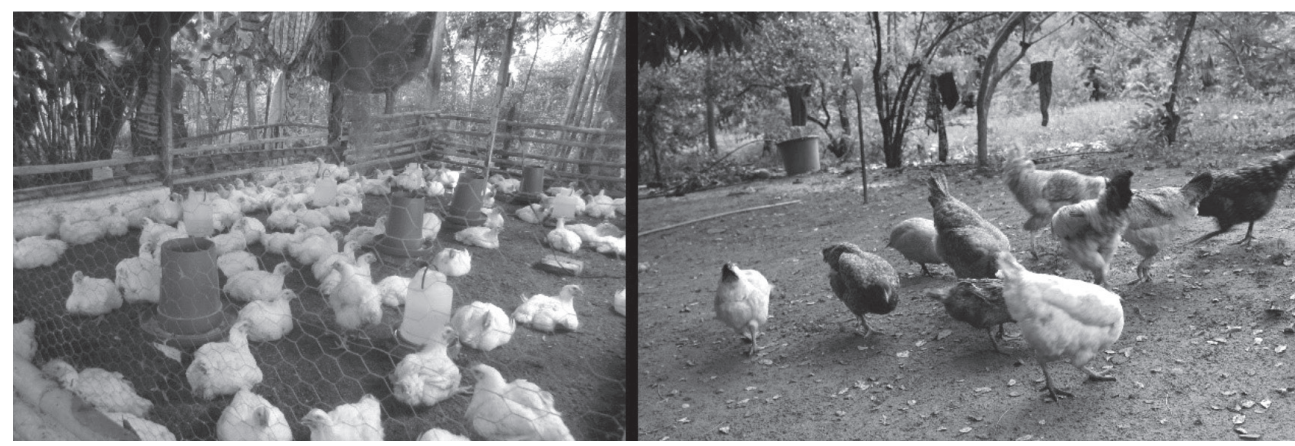

[...] los pollos para vender son alimentados con concentrado, pero si usted los deja para el consumo, entonces usted le sigue dando desperdicios, y sigue dándoles maíz y los deja a patio libre para que ellos se críen como pollos criollos. (Fotosesión 1, Hombre Comunero).

Una mata de plátano con un racimo de ocho gajas, es consecuencia del uso de "triple 15", pero si usted solo le echa el abono propio no le va a dar sino cinco gajas máximo. (Grupo de Discusión 1. Hombre Comunero).

En síntesis, la disponibilidad y acceso de alimentos está asociada con el uso, tenencia y extensión de la tierra, la disposición de recursos hídricos y presencia de especies nativas, relacionados con condiciones climáticas adversas. Otras características intervinientes son invasión de terrenos, construcción de carreteras, explotación minero-energética, uso de semillas transgénicas y agroquímicos. El consumo y utilización de alimentos, depende de los tiempos de siembra y del uso de semillas propias, las cuales pueden ser modificadas por influencia externa, generando una coexistencia de prácticas de producción y consumo de alimentos propios y foráneos, con repercusión importante en la calidad e inocuidad.

\section{Discusión}

Las condiciones de seguridad alimentaria son la disponibilidad, acceso, consumo, aprovechamiento y calidad de los alimentos. Estos aspectos registran particularidades 
en los contextos indígenas en todo el mundo (Peña-Venegas et al., 2009), las cuales están determinadas por condiciones del territorio y por influencia externa.

La disponibilidad y acceso de alimentos asociada con el uso, tenencia y extensión de la tierra, coincide con una de las conclusiones del informe Nacional sobre SAN de pueblos indígenas de Guatemala que dice: "la tenencia de la tierra es uno de los mayores problemas que atenta contra la SAN por que en casos de despojo territorial, la gente sin tierra no puede tener comida, ni sembrar y cosechar" (FAO, n.d.). Desde esta perspectiva, la SAN en comunidades indígenas depende directamente de la tenencia, protección y defensa de los territorios; esto puede explicar las luchas históricas por la reivindicación de derechos territoriales que caracteriza a estos pueblos (GREY; PATEL, 2015).

Adicionalmente, la disponibilidad de alimentos puede verse afectada por la limitada disposición de recursos hídricos, las condiciones climáticas desfavorables y la pérdida de especies nativas. Estos hallazgos son similares a los obtenidos en otros estudios que indican el pobre abastecimiento de agua en territorios indígenas, condición que se contrasta con la indiferencia del Estado para garantizar el acceso a este recurso vital, situación común en países menos desarrollados. En este proceso se evidencia un conflicto entre la soberanía de las comunidades y el papel de los Estado en el manejo de los recursos naturales (BORDIGNON et al., 2015).

Otro factor que afecta la disponibilidad a los alimentos es el cambio climático; éste incide en los ciclos productivos, en las prácticas alimentarias (FAO, 2016; ACHINTE, 2015) y en la pérdida de especies nativas (MADZIMURE et al., 2013). El cambio climático es un tema relevante en el siglo XXI, ejemplo de ello es su inclusión en las agendas políticas internacionales de desarrollo como son los ODS, que instan a avanzar en estrategias de mitigación y acciones para la conservación de la biodiversidad.

Sobre el consumo y utilización de alimentos, estudio realizado en una comunidad Ngäbe costarricense (D’AMBROSIO; PURI, 2016), confirma la preocupación sobre los cambios de dieta de esta comunidad indígena que experimentan rápidas transiciones etnobiológicas, nutricionales y socioeconómicas, mientras se están integrando cada vez más en las sociedades modernizadoras. Otro estudio plantea que el $50 \%$ de los alimentos básicos fueron comprados en tiendas y que la reducción en la producción local de alimentos, puede estar influenciada por nuevas estructuras económicas y agentes relacionados con el Estado y la globalización” (HIGHFIELD, 2017). 
Con relación a la calidad e inocuidad de los alimentos, toman relevancia las percepciones alrededor de los conocimientos ancestrales frente a lo que se considera buena alimentación. Al respecto, estudio realizado con indígenas del Canadá compara los alimentos tradicionales con dietas actuales; los primeros, ricos en nutrientes y los segundos densos en energía y pobres en nutrientes (BHAWRA et al., 2015). Otro estudio en el mismo país, plantea que la "mala alimentación" está asociada al consumo de alimentos altamente procesados, que causan problemas de salud, entre ellos la obesidad en los Indígenas Nunavut Inuit. (EGELAND et al., 2010).

Con base en lo antes expuesto, se confirma la necesidad de introducir alimentos tradicionales cultivados en casa, para la conservación de prácticas alimentarias autóctonas y la protección del conocimiento ancestral en peligro de extinción (D’AMBROSIO; PURI, 2016; HIGHFIELD, 2017). Por otro lado, las nuevas generaciones requieren la implementación de modelos educativos diferenciados basados en el respeto por su tradición y fortalecimiento de la identidad indígena, condiciones recomendadas por estudio realizado en Perú en 2016(HUAMAN, 2016).

\section{Conclusiones}

Las condiciones de seguridad alimentaria de la comunidad indígena La Gabriela están asociadas al territorio y a la influencia externa en las prácticas alimentarias. En general, existe inseguridad alimentaria debido a la aridez de los suelos, insuficiencia de recursos hídricos y cambios climáticos, características que ocasionan pérdida de especies vegetales y animales usados tradicionalmente como fuente de alimentación.

Los cambios de prácticas alimentarias por influencia externa afectan la autonomía y generan dependencia de cadenas comerciales fortalecidas por el libre mercado. Es preciso formular políticas que fortalezcan las creencias, costumbres y tradiciones ancestrales, lo cual requiere mayor atención por parte de las entidades estatales y los gobiernos. ${ }^{1}$

\section{Agradecimientos}

Apreciamos el compromiso y acogida de la comunidad del Resguardo Indígena Tama Páez La Gabriela, por compartir su sabiduría y vivencias. Agradecemos también al Consejo Regional Indígena del Huila- CRIHU y al Cabildo indígena universitario 
de la Universidad Surcolombiana (CIUSCO), por su apoyo y acompańamiento. Este proyecto fue financiado por la Universidad Surcolombiana.

\section{Referencias}

ACHINTE, A. A. Sabor, poder y saber: Comida y tiempo en los valles afroandinos del Patía y Chota-Mira (Universidad). Colombia: Popayán, 2015.

AMEZCUA, M.; TORO, A. G. Los Modos de Análisis en Investigación Cualitativa en Salud: perspectiva crítica y reflexiones en voz alta, v. 76, n. 5, p. 423-436, 2002.

ANTICONA, D. M.; GAVIDIA, S. O.; TELLO, A. M. Estudio etnográfico focalizado sobre la alimentación, el crecimiento y el desarrollo del niño en comunidades rurales de Huancavelica. In: ORGANIZACIÓN PANAMERICANA DE LA SALUD. Investigaciones Operativas en Salud y Nutrición de la Niñez en el Perú. Lima: OPS, 2008. p. 227-237.

ARIAS, M. et al. Nutritional state associated with social determinants in Arhuaco children aged less than 5 years-old. Revista de Salud Pública, v. 15, n. 4, p. 613-625, 2013.

BARRETO, C. T. G.; CARDOSO, A. M.; COIMBRA JR, C. A. Nutritional status of Guarani indigenous children in the States of Rio de Janeiro and São Paulo, Brazil. Cadernos de Saúde Pública. Rio de Janeiro, v. 30, n. 3, p. 657-662, 2014.

BHAWRA, J. et al. Community perspectives on food insecurity and obesity: focus groups with caregivers of Métis and off-reserve first nations children. International Journal for Equity in Health, v. 14, n. 1, p. 96, 2015.

BORDIGNON, M.; GRECO, R.; LEPORE, G. Water Grabbing and Water Rights: indigenous "sovereignty" vs State "sovereignty". In: JACUR, F. R. et al. (Eds.). Natural Resources Grabbing: an International Law Perspective. Boston: Brill, 2015.

BRANT, M. Ethics of aboriginal research. Journal of Aboriginal Health, n. 1, p. 98-114, 2004.

CALLEJO, J. Técnicas grupales. In: . Introducción a las técnicas de investigación social. Madrid: Editorial Universitaria Ramon Areces, 2009.

COIMBRA JR., C.; COIMBRA, E. A. Health and indigenous peoples in Brazil: reflections based on the First National Survey of Indigenous People's Health and Nutrition. Cadernos de Saúde Pública. Rio de Janeiro, v. 30, n. 4, p. 855-859, 2014.

CONSEJO DE ORGANIZACIONES INTERNACIONALES DE LAS CIENCIAS MÉDICAS (CIOMS). Organización Mundial de la Salud (OMS). Pautas éticas Internacionales para la investigación biomédica en seres humanos. CIOMS, 2002.

CONSEJO NACIONAL DE POLÍTICA ECONÓMICA. Departamento Nacional de Planeación. Politica Nacional de Seguridad Alimentaria y Nutricional (PSAN). Bogotá, 2007. 
D'AMBROSIO, U.; PURI, R. K. Foodways in transition: food plants, diet and local perceptions of change in a Costa Rican Ngäbe community. Journal of Ethnobiology and Ethnomedicine, v. 12, n. 3, p. 1-32, 2016.

EGELAND, G. M. et al. Food insecurity among Inuit preschoolers: Nunavut Inuit Child Health Survey, 2007-2008. Canadian Medical Association Journal, v. 182, n. 3, p. 243-248. 2010.

GREY, S.; PATEL, R. Food sovereignty as decolonization: some contributions from Indigenous movements to food system and development politics. Agriculture and Human Values, v. 32, n. 3, p. 431-444, 2015.

HIGHFIELD, J. B. Food and foodways in African narratives: community, culture, and heritage. California, San Diego: Routledge, 2017.

HUAMAN, E. S. Tuki Ayllpanchik (our beautiful land): Indigenous ecology and farming in the Peruvian highlands. Cultural Studies of Science Education, v. 11, n. 4, p. 1.135-1.153, 2016. HUERTAS, E. B.; VIGIER, F. J. El grupo de discusión como técnica de investigación en la formación de traductores: dos casos de su aplicabilidad. Entreculturas, n. 2, p. 181-196, 2010.

IPS-I CRIHU. Clasificación nutricional en menores de 5 años de las comunidades indígenas. Municipios de Iquira, La Plata y La Argentina, 2013.

MADZIMURE, J. et al. Potential for using indigenous pigs in subsistence-oriented and market-oriented small-scale farming systems of Southern Africa. Tropical Animal Health and Production, v. 45, n. 1, p. 135-42, 2013.

COLOMBIA. Ministerio de Salud. Resolución n. 008430 de 1993. p. 3-6, octubre. 1993.

MORAN, M. Hambre y seguridad alimentaria, desarrollo sostenible. Nueva York: United Nations, 2015.

NACIONES UNIDAS. Objetivos de Desarrollo del Milenio. Nueva York: United Nations, 2015 (Informe 2015).

ORGANIZACIÓN DE LAS NACIONES UNIDAS PARA LA ALIMENTACIÓN Y LA AGRICULTURA (FAO). Programa Especial para la Seguridad Alimentaria (PESA). Nueva York: United Nations, February 2, 2018.

- Cambio climático y seguridad alimentaria y nutricional América Latina y el Caribe. Nueva York: United Nations, 2016

. Departamento para la Prosperidad Social. Comida, territorio y memoria: situación alimentaria de los pueblos indigenas colombianos. Nueva York: United Nations, 2015.

Informe Nacional: Seguridad Alimentaria y Nutricional y Pueblos Indígenas en Guatemala. Nueva York: United Nations, 2014. 
PENAA-VENEGAS, C. P. et al. (Eds.). Seguridad alimentaria en comunidades indigenas del Amazonas: ayer y hoy. Colombia: Instituto Sinchi, 2009.

QUINN, M. Qualitative research and evaluation methods $3^{\text {rd }}$ ed. United States of America. New York: Sage, 2002.

RAMOS, C. et al. Factores sociales y culturales que influyen en el estado nutricional de los nińos menores de tres ańos de edad en comunidades pobres de la provincia de Satipo. Investigación operativa. OPS, 2002.

REPÚBLICA DE COLOMBIA; CONSEJO NACIONAL DE POLÍTICA ECONÓMICA SOCIAL (CONPES). Politica Nacional de seguridad alimentaria y nutricional (PSAN). Bogotá: CONPES, 2008.

TELLO, A. M.; MIRANDA, L. A.; ESPINOZA, J. M. La salud y nutrición infantil en comunidades indígenas achuares, urarinas y quichuas de la región Loreto, año 2006. In: ZALDÍVAR, H. P. (Ed.). Investigaciones operativas en salud y nutrición de la niñez en el Perú, $\mathrm{n}$. 61. Lima: Ministerio de la Salud, 2006.

WANG, C.; BURRIS, M. A. Empowerment through Photo Novella: portraits of participation. Health Education \& Behavior, v. 21, n. 2, p. 171-186, 1994.

\section{Nota}

${ }^{1}$ J. C. C. Farfán realizó la formulación del proyecto, validación de instrumentos, recolección, análisis de la información y escritura del artículo científico. D. A. Torres realizó el apoyo en la recolección, análisis de la información y escritura del artículo científico. M. N. P. Gómez fue responsable por la formulación del proyecto, validación de instrumentos, recolección y análisis de la información y escritura del artículo científico. M. P. Q. Tamayo realizo la formulación de la propuesta, validación de instrumentos, recolección y análisis de la información. 


\section{Abstract}

\section{Conditions of food security in an indigenous community of Colombia}

Food security is achieved when all people, at all times, have physical and economic access to sufficient food, safe and nutritious, to meet their dietary needs and preferences, in order to lead an active and healthy life. Objective: To explore conditions of food security in the community members of the La Gabriela Indigenous Reserve. Method: Qualitative study, of exploratory scope, that applied the techniques of photo-voice and discussion groups. Content analysis was carried out. Results: The food security conditions of the La Gabriela indigenous community are associated to the territory and food practices modified by external influence, which affects their autonomy generating dependence on commercial chains stimulated by the free market. It is necessary to formulate policies that strengthen ancestral beliefs, customs and traditions, which requires greater attention on the part of state entities and governments.

Keywords: Food security; conditions; feeding; indigenous community; food production. 


\section{Resumo}

\section{Condiçôes de segurança alimentar em uma comunidade indígena da Colômbia}

A segurança alimentar é alcançada quando todas as pessoas, em todos os momentos, têm acesso físico e econômico a alimentos suficientes, seguros e nutritivos, para satisfazer suas necessidades e preferências alimentares, a fim de levar uma vida ativa e saudável. Objetivo: Explorar as condições de segurança alimentar dos membros da comunidade da Reserva Indígena La Gabriela. Método: Estudo qualitativo, de abrangência exploratória, que aplicou as técnicas de foto-voz e grupos de discussão. Realizou-se análise de conteúdo. Resultados: As condiçóes de segurança alimentar da comunidade indígena La Gabriela estão associadas ao território e às práticas alimentares modificadas por influência externa, o que afeta sua autonomia, gerando dependência de cadeias comerciais estimuladas pelo mercado livre. É necessário formular políticas que fortaleçam crenças, costumes e tradiçóes ancestrais, o que requer maior atenção por parte das entidades e governos estaduais.

> Palavras-chave: segurança alimentar; condições; alimentação; comunidade indígena; produção de alimentos. 